\title{
Outlier Detection and Correction for the Deviations of Tooth Profiles of Gears
}

\author{
Han Lianfu $^{1,2}$, Fu Changfeng ${ }^{2}$, Wang Jun ${ }^{1}$, Tang Wenyan ${ }^{1}$ \\ ${ }^{1}$ Institute of Precision Instrument, School of Electrical Engineering and Automation, Harbin Institute of Technology, \\ West Dazhi Street 92, 150001, Harbin, China, \\ e-mail: lianfuhan@hit.edu.cn,wangjun@hit.edu.cn, tangwy@hit.edu.cn \\ ${ }^{2}$ School of Electronic Science, Northeast Petroleum University, Fazhan street 199, 163318, Daqing, China, \\ e-mail:fuchangfeng8001@126.com
}

\begin{abstract}
To decrease the influence of outlier on the measurement of tooth profiles, this paper proposes a method of outlier detection and correction based on the grey system theory. After studying the characteristics of outliers from the deviations of tooth profiles, this paper proposes a preprocessing method for the modeling data which include abnormal value, and establishes an outlier detection and correction model for the deviations of tooth profiles. Simulation results show that the precision of ONDGM(1,1)(one order and one variable non-homogenous discrete grey model whose outlier is processed by the preprocessing method proposed in this paper) is higher than that of $\operatorname{NDGM}(1,1)($ one order and one variable non-homogenous discrete grey model), and the ONDGM(1,1) is more suitable than the NDGM(1,1) for dealing with the outliers from the deviations of tooth profiles. The experiment results show that the outlier detection and correction model detects and corrects the outliers from the deviations of tooth profiles, and the correction value of the outlier is basically in accordance with the actual deviation. Therefore, the method of outlier detection and correction decreases the influence of outlier and improves the precision in the measurement of tooth profiles.
\end{abstract}

Keywords: Tooth profile, outlier detection, grey system theory, gear measuring center

\section{INTRODUCTION}

$\mathrm{G}$ EARS ARE widely used in mechanical manufacturing, and the quality of tooth profiles affects gear vibration and noise [1-2]. The measurement of tooth profiles is a difficult measurement item, and now tooth profiles are usually measured by gear measuring center [3-5]. Gear measuring center is a kind of efficient precision measuring instrument, but outliers easily cause sudden measurement environment changes in the measurement of tooth profiles, such as the effect of mechanical vibration on the measuring head and the impact of the sharp current on the data acquisition card [6-8]. The outliers lead to the abnormal data and decrease the measurement precision of tooth profiles, so the outliers must be removed as soon as they are detected [9-11].

Processing of outlier from tooth profile can be regarded as a kind of filter. Kalman filter, particle filter, adaptive filter and wiener filter are widely used in the measurement signal of tooth profile. Models of these filters are very complex and their calculation is very large. Models of these filters need large modeling data, and some of them require that modeling data conform to the Gaussian distribution or other known distribution. However, the deviations of tooth profiles do not conform to the known distribution and sometimes there is only little modeling data. The methods for detecting and correcting outliers mainly include $3 \alpha$ criterion, Chauvenet criterion, Grubbs criterion, Dixon criterion, and etc. [12]. These methods require that the deviations conform to the Gaussian distribution or other known distribution, but the deviations of tooth profiles do not conform to the known distribution. Recently, more and more researchers have been focusing on the method of outlier detection and correction based on the grey system theory, because this method does not require the data to conform to the known distribution [13-15]. Meng proposes an outlier detection method based on the $\operatorname{GM}(1,1)$ model to detect the abnormal data during dynamic measurement of discontinuous surfaces [16]. Shi improves the $\operatorname{GM}(1,1)$ model (one order and one variable grey model) and utilizes it in the detection of the gross error for hob edge [17]. Wang proposes an outlier detection method based on the grey system theory for the outlier of the measurement of the work piece [18]. Wang develops an outlier processing based on the grey system theory for non-diffracting beam triangulation measurement system [12]. The previous methods can detect the outlier, however, the modeling data of the previous methods is the original sequence which includes outliers. The outliers of the original sequence produce the abnormal data in the predictive sequence, resulting in the fact that the outlier cannot be corrected by the abnormal predictive value. This is in agreement with the conclusions of Ke [19], but Ke does not carry out further studies on how to preprocess the original data.

To address the problem, this paper proposes a preprocessing method for the abnormal modeling data according to the characteristics of the outliers from the tooth profiles and the features of $\operatorname{NDGM}(1,1)$ (one order and one variable non-homogenous discrete grey model) and establishes a model for outlier detection and correction. The experiment results show that the model detects and corrects the outliers of the deviations of tooth profiles, and improves the precision in the measurement of tooth profiles. 


\section{MATHEMATICAL MODEL FOR OUTLIER DETECTION AND CORRECTION}

\subsection{Characteristics of the outliers from tooth profiles}

Fig.1. is a typical deviation curve of tooth profiles containing the outliers in the deviations. To display the deviation clearly, the deviations are enlarged 1000 times. As shown in Fig.1., the characteristics of the deviation curve of tooth profiles are as follows.

1). Irregularity. The deviations of tooth profiles do not conform to the known distribution.

2). Isolated point. No continuous outliers exist in the deviation curve of tooth profiles, which is basically in accordance with the conclusion made by Lou and Wang [20].

The grey system theory is proper for detecting and correcting outliers, because the deviations of tooth profiles do not conform to the known distribution and the grey system theory does not require the data to conform to any known distribution. However, the precision of the correct value is affected by the outlier of the deviations of tooth profiles if the original deviations of tooth profiles are directly used to establish the outlier detection and correction model. Therefore, the original deviation sequence including the outlier should be preprocessed. Since the outlier from the deviations of tooth profiles is an isolated point, the preprocessing method for the deviations of tooth profiles, which includes the isolated outlier, is introduced.

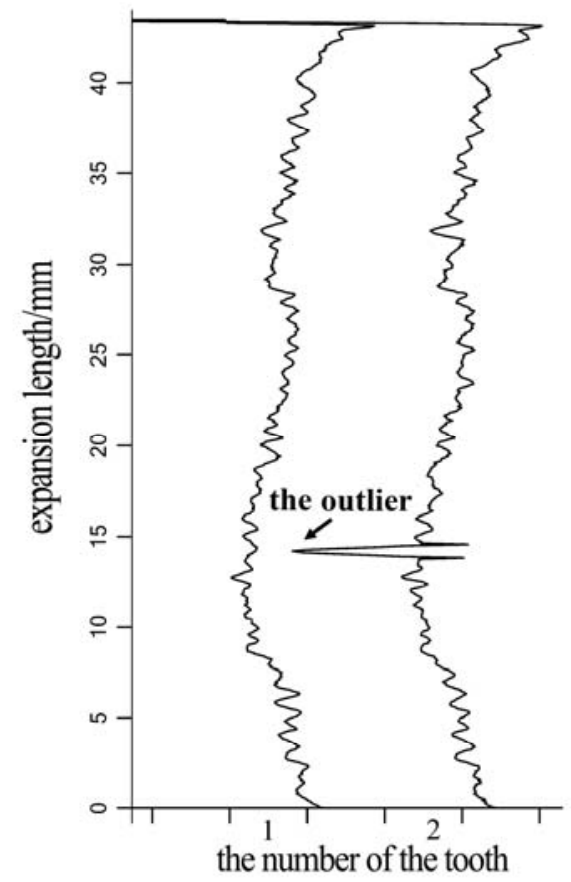

Fig.1. Deviation curve of tooth profiles with outlier

\subsection{The preprocessing method for the original deviations of tooth profiles}

\subsubsection{The preprocessing method}

$\mathrm{X}^{(0)}=\left\{x^{(0)}(1), x^{(0)}(2), \ldots, x^{*(0)}(\mathrm{m}), \ldots, x^{*(0)}(\mathrm{k}), \ldots, x^{(0)}(\mathrm{n}-1)\right.$, $\left.x^{(0)}(\mathrm{n})\right\}$ is the original deviation sequence with outlier, and $x^{*^{(0)}}(\mathrm{m})$ and $x^{*(0)}(\mathrm{k})$ are the outliers. The precision of discrete grey forecasting model is higher than that of the $\operatorname{GM}(1,1)$ model and the $\operatorname{NDGM}(1,1)$ model is more suitable to deal with the actual data than the $\operatorname{DGM}(1,1)$ model $[20$ 24]. Therefore, this paper focuses only on the preprocessing method for the modeling data of the $\operatorname{NDGM}(1,1)$ model.

Definition 1. Assume that sequence $\mathrm{X}^{(0)}=\left\{x^{(0)}(1)\right.$, $\left.x^{(0)}(2), \ldots, x^{*(0)}(\mathrm{m}), \ldots, x^{*(0)}(\mathrm{k}), \ldots, x^{(0)}(\mathrm{n}-1), x^{(0)}(\mathrm{n})\right\}$ is the original sequence, and sequence $\hat{X}^{(0)}=\left\{\hat{x}^{(0)}(1), \hat{x}^{(0)}(2), \ldots, \hat{x}^{(0)}(n), \hat{x}^{(0)}(n-1)\right\} \quad$ is the predictive sequence achieved by the $\operatorname{NDGM}(1,1)$ model, then the equation

$$
\hat{\gamma}^{(0)}(k)=\frac{\hat{x}^{(0)}(k+1)-\hat{x}^{(0)}(k)}{\hat{x}^{(0)}(k)-\hat{x}^{(0)}(k-1)}
$$

is called the adjacent variance ratio of the $\operatorname{NDGM}(1,1)$ model.

Theorem 1. The adjacent variance ratio of the $\operatorname{NDGM}(1,1)$ model is a constant, and $\hat{\gamma}^{(0)}(k)=\beta_{1}$.

Proof. According to the NDGM $(1,1)$ model,

$$
\left\{\begin{aligned}
\hat{x}^{(1)}(k+1) & =\beta_{1} \hat{x}^{(1)}(k)+\beta_{2} k+\beta_{3} \\
\hat{x}^{(1)}(0) & =x^{(0)}(1)+\beta_{4}
\end{aligned}\right.
$$

$\hat{x}^{(0)}(k+1)$ is expressed as $(3)$

$$
\begin{aligned}
\hat{x}^{(1)}(k+1) & =\beta_{1}^{k}\left(x^{(0)}(1)+\beta_{4}\right) \\
& +\beta_{2} \sum_{j=1}^{k} j \beta_{1}^{k-j}+\frac{1-\beta_{1}^{k}}{1-\beta_{1}} \beta_{3}
\end{aligned}
$$

According to (3), the following is obtained.

$$
\begin{aligned}
\hat{x}^{(1)}(k) & =\beta_{1}^{k-1}\left(x^{(0)}(1)+\beta_{4}\right) \\
& +\beta_{2} \sum_{j=1}^{k-1} j \beta_{1}^{k-1-j}+\frac{1-\beta_{1}^{k-1}}{1-\beta_{1}} \beta_{3}
\end{aligned}
$$

$\hat{x}^{(0)}(k)$ is expressed as (5).

$$
\hat{x}^{(0)}(k)=\hat{x}^{(1)}(k+1)-\hat{x}^{(1)}(k)
$$

Substitute $\hat{x}^{(1)}(k+1)$ and $\hat{x}^{(1)}(k)$ into (5), (6) is obtained.

$$
\begin{aligned}
\hat{x}^{(0)}(k)= & \beta_{1}^{k-1}\left\{\beta_{1}\left[x^{(0)}(1)+\beta_{4}\right]+\beta_{3}-\frac{\beta_{1} \beta_{2}}{1-\beta_{1}}\right\} \\
& +\frac{\beta_{2}}{1-\beta_{1}}
\end{aligned}
$$




$$
\text { Let }\left\{\begin{array}{l}
m=\beta_{1}\left[x^{(0)}(1)+\beta_{4}\right]+\beta_{3}-\frac{\beta_{1} \beta_{2}}{1-\beta_{1}} \\
n=\frac{\beta_{2}}{1-\beta_{1}}
\end{array}\right.
$$

According to (6) and (7), the following is obtained.

$$
\begin{aligned}
\hat{\gamma}^{(0)}(k) & =\frac{\hat{x}^{(0)}(k+1)-\hat{x}^{(0)}(k)}{\hat{x}^{(0)}(k)-\hat{x}^{(0)}(k-1)} \\
& =\frac{\left(m \beta_{1}^{k-1}+n\right)-\left(m \beta_{1}^{k-2}+n\right)}{\left(m \beta_{1}^{k-2}+n\right)-\left(m \beta_{1}^{k-3}+n\right)} \\
& =\beta_{1}
\end{aligned}
$$

According to Theorem 1, the adjacent variance ratio of the $\operatorname{NDGM}(1,1)$ model is $\beta_{1}$. The original data sequence is suitable to be dealt with by the $\operatorname{NDGM}(1,1)$ model, so the adjacent variance ratio is in the range of $\left(\beta_{1}-\Delta \beta_{1}, \beta_{1}+\Delta \beta_{1}\right)$, where $\Delta \beta_{1}$ is a minor value. Therefore, we can detect the outlier of the original data sequence by detecting the outlier of the adjacent variance ratio of the original data sequence.

The outlier of the adjacent variance ratio of the original data sequence is preliminarily detected using the method of Wang [16]. The measurement system is considered as a grey system and the deviations of tooth profiles are grey numbers, so the adjacent variance ratios of the deviations are also grey numbers. $\hat{\gamma}^{(0)}$ is sorted in ascending order, and the new ascending sequence is named as $\xi^{(0)}$. $\xi^{(1)}$ can be attained by (9).

$$
\xi^{(1)}(k)=\sum_{i=1}^{k} \xi^{(0)}(i)
$$

$\xi^{(1)}(k)$ (line 1) should be in the particular area between line 3 and the 2-segment line 2, as shown in Fig.2. The upper side of the grey area is determined based on the reference line (line 3). Line 3 is expressed as (10).

$$
\xi_{\max }^{(1)}(k)=\frac{1}{n} \xi^{(1)}(n) k=\left[\frac{1}{n} \sum_{i=1}^{n} \xi^{(0)}(i)\right] k=\bar{\xi}_{k}
$$

where, $k=1,2,3, \ldots, n$, and $\bar{\xi}$ is the arithmetic mean of the adjacent variance ratios of the deviations.

The lower side of the grey area is determined based on the 2 -segment line (line 2). Line 2 is expressed as (11).

$$
\xi_{\min }^{(1)}(k)= \begin{cases}\left(\bar{\xi}-\frac{h \Delta_{\max }}{p}\right) k & (1 \leq k \leq p) \\ \bar{\xi} k-\frac{h \Delta_{\max }}{n-p}(n-k) & (\mathrm{p}<k \leq n)\end{cases}
$$

where, $h$ is taken as 3.75 and $p$ is expressed as (12) [18].

$$
p= \begin{cases}\frac{n}{2} & (\text { even } n) \\ \frac{n+1}{2} & (\text { odd } n)\end{cases}
$$



Fig.2. Accumulation data curve of the deviations of tooth profiles.

$\xi^{(1)}(k)$ is in the grey area if $\xi^{(1)}(k)$ meets (13), so $\xi^{(1)}(k)$ is not the outlier. Otherwise, $\xi^{(1)}(k)$ is outside of the grey area, so it is the outlier.

$$
\xi_{\min }^{(1)}(k)<\xi^{(1)}(k)<\xi_{\max }^{(1)}(k)
$$

According to $(1), \mathrm{x}^{*(0)}(\mathrm{m})$ is the abnormal value if $\xi^{(1)}(m), \quad \xi^{(1)}(m+1), \xi^{(1)}(m+2)$ are abnormal. The outlier of the original data sequence is preliminarily detected by the preceding method, and the preliminary outlier is corrected by (14) based on the definition of the adjacent variance ratio.

$$
\breve{x}^{(0)}(m)= \begin{cases}\frac{(1+c) x^{(0)}(2)-c x^{(0)}(3)}{c} & (m=1) \\ (1+c) x^{(0)}(m-1)+x^{(0)}(m-2) & (m=\mathrm{n}) \\ \frac{x^{(0)}(m+1)+c x^{(0)}(m-1)}{1+c} & (\text { others })\end{cases}
$$

where, $\mathrm{c}$ is the average of the adjacent variance ratio of the original data sequence which does not include the outliers.

\subsubsection{Simulation}

To prove the feasibility of the proposed preprocessing method in the modeling data, a simulation is carried out. The modeling data $X^{(0)}=\{3.0430,3.0145,3.2297,3.3106$, 3.5751, 2.1, 3.8370, 3.9965, 4.2424, 4.3983, 4.7465, 4.9828, $5.3216,5.5951\}$ is achieved by (15). 


$$
x^{(0)}(k)=e^{0.1 k}+2+\operatorname{rand}(k)
$$

where, $\operatorname{rand}(k)$ is a grey number, $\otimes \operatorname{rand}(k) \subset[-0.04,0.04]$ and $x^{(0)}(6)$ is the artificially introduced outlier. The $\operatorname{NDGM}(1,1)$ model whose modeling data is processed by the preprocessing method proposed in this paper is noted as the $\operatorname{ONDGM}(1,1)$ model. Fig.3. and Fig.4. show the simulations of the $\operatorname{NDGM}(1,1)$ model and the $\operatorname{ONDGM}(1,1)$ model.

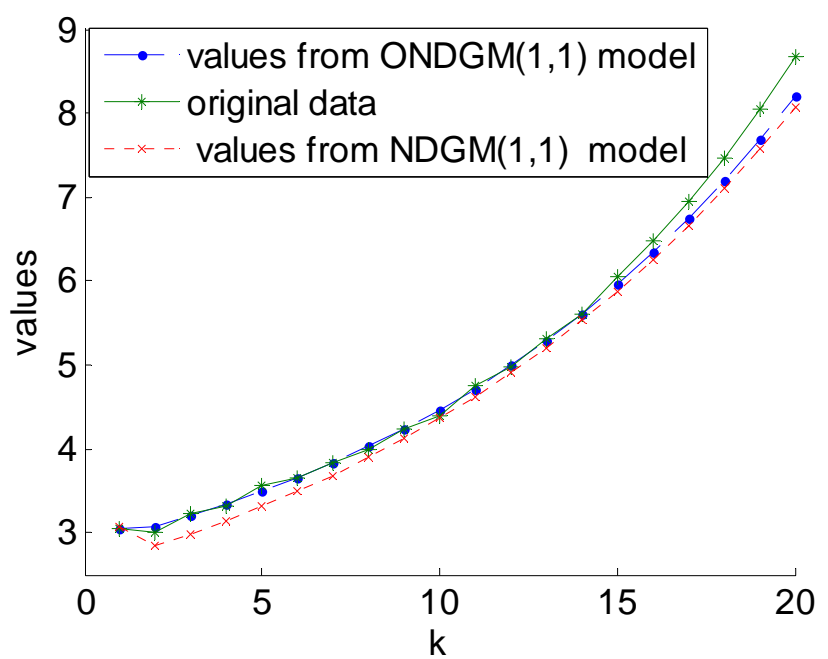

Fig.3. Comparison of prediction performance

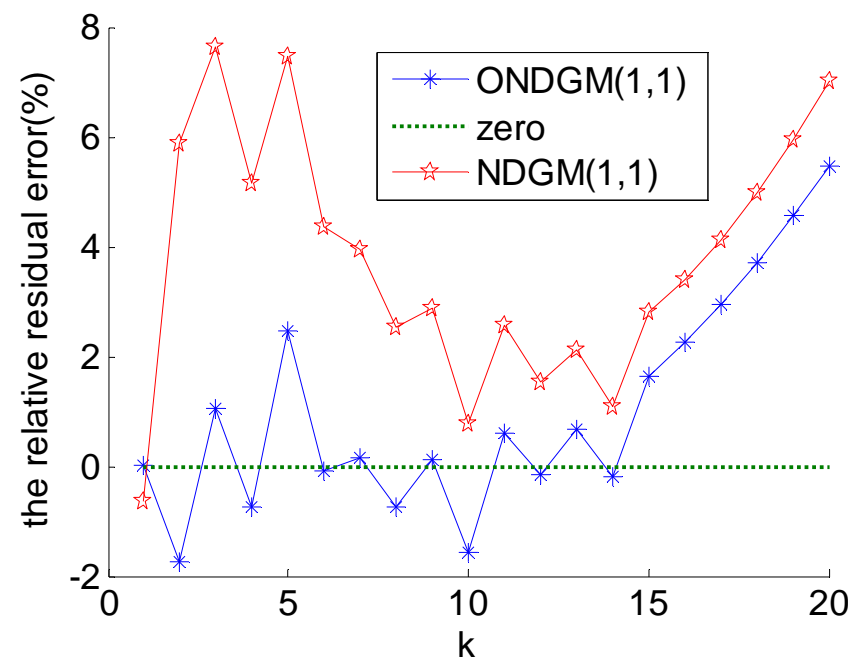

Fig.4. Comparison of prediction data error

As shown in Fig.3., the values from the $\operatorname{NDGM}(1,1)$ model become smaller than the original data for the outlier $x^{(0)}(6)$, while the values from the $\operatorname{ONDGM}(1,1)$ model are not affected by the outliers. As shown in Fig.4., any relative residual error of the $\operatorname{NDGM}(1,1)$ model is larger than that of the ONDGM(1,1) model. The relative residual errors of the $\operatorname{NDGM}(1,1)$ model are almost positive, while the relative residual errors of the $\operatorname{ONDGM}(1,1)$ model are symmetrical to the center zero, which is in accordance with the actual distribution of data. The relative errors of $x^{(0)}(2), x^{(0)}(3), x^{(0)}(4)$ and $x^{(0)}(5)$ from the $\operatorname{NDGM}(1,1)$ model become larger for the outlier $x^{(0)}(6)$ compared to those from the $\operatorname{ONDGM}(1,1)$ model. The maximum relative residual error of the $\operatorname{NDGM}(1,1)$ model is $7.9 \%$, while the maximum relative residual error of the $\operatorname{ONDGM}(1,1)$ model is $2.7 \%$.

\subsection{The model of outlier detection and correction}

The deviation of any point at the tooth profiles is $x^{(0)}(k)$ and the prediction value of it is $\hat{x}^{(0)}(k)$, so the residual error $e^{(0)}(k)$ is expressed as (16).

$$
e^{(0)}(k)=x^{(0)}(k)-\hat{x}^{(0)}(k)
$$

The standard deviation is expressed as (17).

$$
S=\sqrt{\frac{\sum_{k=1}^{n} e^{2}(k)}{n-1}}
$$

If any value $x^{(0)}(k)$ meets (18), the value at this point is called outlier, and then the outlier is corrected by (19).

$$
\left|e^{(0)}(k)\right|>\kappa S
$$

$$
x^{(0)}(k)=\hat{x}^{(0)}(k)
$$

where $\kappa$ is the recognition coefficient related to the addendum circle diameter and the quality of tooth profiles. $\kappa$ can be obtained by experience, and it is determined to be 2.7 in the outlier detection and correction of the deviations of tooth profiles [17]. The automatic detection of outlier is achieved according to (18), and the outliers are corrected according to (19).

\section{EXPERIMENT}

Fig.5. shows the measurement equipment. L45-type CNC gear measuring center jointly developed by our institute and Harbin Measuring \& Cutting Tool Group Co., Ltd is used in the experiment and the work piece to be measured is an involute master of gear. Parameters of the involute master are as follows:

$r_{b}$ (radius of the base circle) is $59.975 \mathrm{~mm}$;

$F_{\alpha}$ (total profile deviation) is- $1.3 \mu \mathrm{m}$;

$f_{f_{\alpha}}$ (profile form deviation) is $-1.3 \mu \mathrm{m}$;

$\pm f_{H \alpha}$ (profile slope deviation) is $-0.5 \mu \mathrm{m}$.

The involute master of gear is clamped on the platform of the gear measuring center. The involute master is measured for many times in the same measurement condition until the deviation curve with sharp pulse is found. Fig.6. shows the deviation curves with the outlier. 


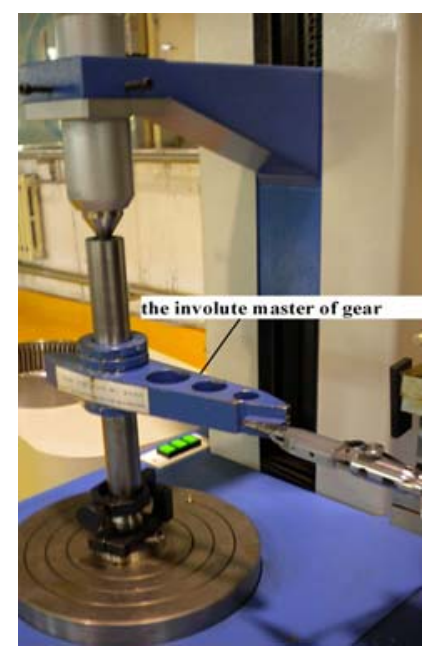

Fig.5. Experimental equipment

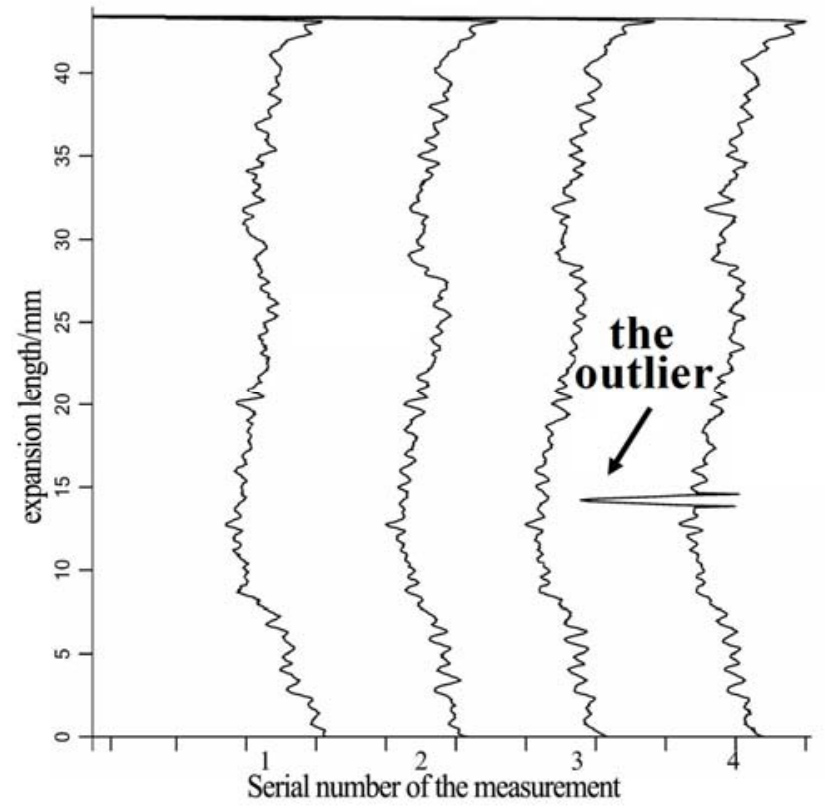

Fig.6. Deviation curves of tooth profiles with outlier

\section{RESULTS AND DISCUSSION}

Fig.6. shows four deviation curves. The deviation distance from the vertical line in every curve stands for the deviation of tooth profiles. The axis of ordinates stands for expansion length, and the unit is $1 \mathrm{~mm}$. The axis of abscissas stands for tooth profiles deviation, and the unit of profile deviation is 1 $\mu \mathrm{m}$. The deviation curves of tooth profiles are straight lines if there are no deviations in the profiles. To display the deviation clearly, the deviations are enlarged 1000 times in Fig.6. Compared with the other curves, a sharp pulse appears at the position where expansion length is about 14.2 $\mathrm{mm}$ in the fourth curve. The deviation curve should be continuous in a way. Therefore, the sharp pulse in the fourth curve is an outlier. The $\operatorname{ONDGM}(1,1)$ model and the $\operatorname{NDGM}(1,1)$ model are utilized to achieve predictive data of the deviations. Then the model for outlier detection and correction is established with the predictive data. The model of outlier detection and correction, whose modeling data achieved by the $\operatorname{NDGM}(1,1)$ model is denoted as the DC-
NDGM model, and the model for outlier detection and correction, whose modeling data achieved by the ONDGM $(1,1)$ model is denoted as the DC-ONDGM model.

Fig.7(a). shows the deviation curves of tooth profiles processed by the DC-NDGM model, and Fig.7(b). shows the deviation curves of tooth profiles processed by the DCONDGM model. The detection and correction results near the outliers are shown in Table 1 . The value of the outlier in Fig.7(a). is smaller than that in Fig.6., indicating that the DC-NDGM model can detect the outlier of tooth profiles, but the value of the outlier in Fig.7(a). is still larger than the actual deviation. The actual deviation of any point of the tooth profile is the average value of 30 times measurement results. The outlier disappears in Fig.7(b). compared to that in Fig.6., and the correction value approximates to the actual deviation compared to the other curves. According to Table 1 , the maximum error attained by DC-ONDGM model is 0.1 $\mu \mathrm{m}$ whereas the maximum error attained by the DC-NDGM model is $1.6 \mu \mathrm{m}$. The correction value of the outlier attained by the DC-ONDGM model is $-0.8 \mu \mathrm{m}$, which approximates to the actual deviation $-0.9 \mu \mathrm{m}$. The correction value of outlier attained by the DC-NDGM model is $-2.5 \mu \mathrm{m}$, which is different from the actual deviation. The DC-NDGM values near the outlier become abnormal in Table 1. and Fig.7(a)., because the predictive value is affected by the outlier of the original data sequence.

Table 1. Results of DC-NDGM and DC-ONDGM near the outlier $(\mu \mathrm{m})$

\begin{tabular}{cccc}
\hline $\begin{array}{c}\text { Measurement } \\
\text { value }\end{array}$ & $\begin{array}{c}\text { DC- } \\
\text { ONDGM }\end{array}$ & $\begin{array}{c}\text { Actual } \\
\text { deviation }\end{array}$ & $\begin{array}{c}\text { DC- } \\
\text { NDGM }\end{array}$ \\
\hline-0.8 & -0.8 & -0.8 & -0.9 \\
-0.9 & -0.9 & -0.9 & -1.4 \\
-4.4 & -0.9 & -0.8 & -2.8 \\
-1.1 & -0.7 & -0.7 & -1.7 \\
-0.8 & -0.8 & -0.8 & -0.7 \\
\hline
\end{tabular}

The tooth profile is evaluated according to the four deviation curves. Table 2. lists the evaluation results of original deviations of tooth profiles, evaluation results processed by the DC-ONDGM model and evaluation results processed by the DC-NDGM model. As indicated in Table 2., the original evaluation results deviate from the actual deviation. The actual evaluation value of $F_{\alpha}$ is -1.3 $\mu \mathrm{m}$, but the original evaluation value is $-4.4 \mu \mathrm{m}$. The actual evaluation value of $f_{f_{\alpha}}$ is $-1.3 \mu \mathrm{m}$, but the original evaluation value is $-3.6 \mu \mathrm{m}$. The actual evaluation value of $\pm f_{H \alpha}$ is $-0.5 \mu \mathrm{m}$, but the original evaluation value is -1.8 $\mu \mathrm{m}$. This leads to the wrong grade accuracy of tooth profiles. The evaluation result processed by the DC-NDGM model is near to the actual deviation. For example, $F_{\alpha}$ decreases from $-4.4 \mu \mathrm{m}$ to $-2.8 \mu \mathrm{m}$, but it has a deviation from the actual result and still causes the wrong grade accuracy of tooth profiles. The evaluation value processed by the DC-ONDGM model approximates to the actual deviation, and it does not affect the evaluation of grade accuracy. 


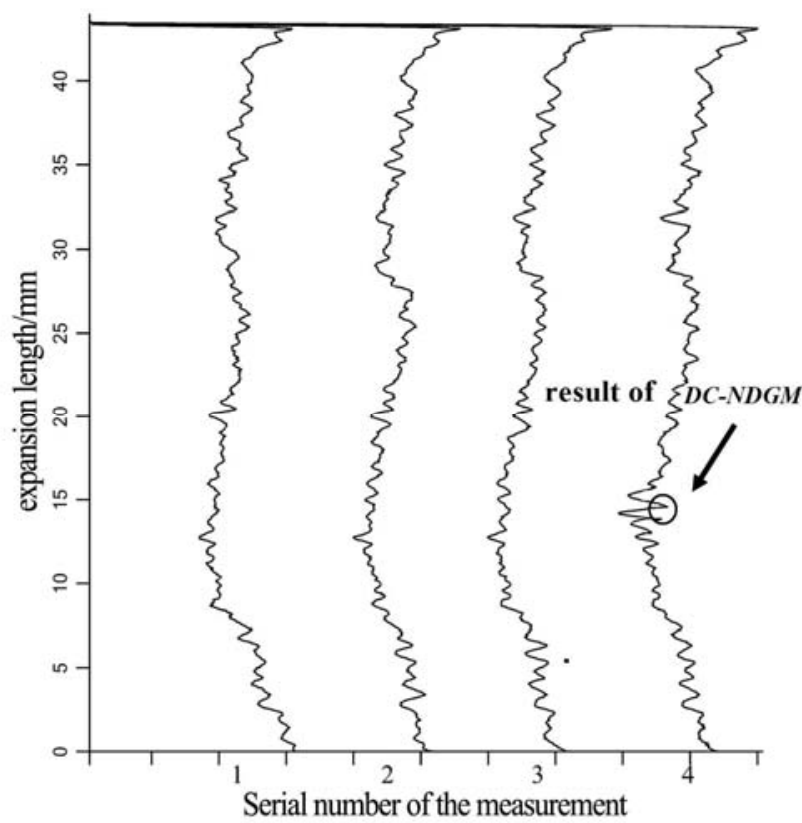

(a). deviation curve processed by DC-NDGM model

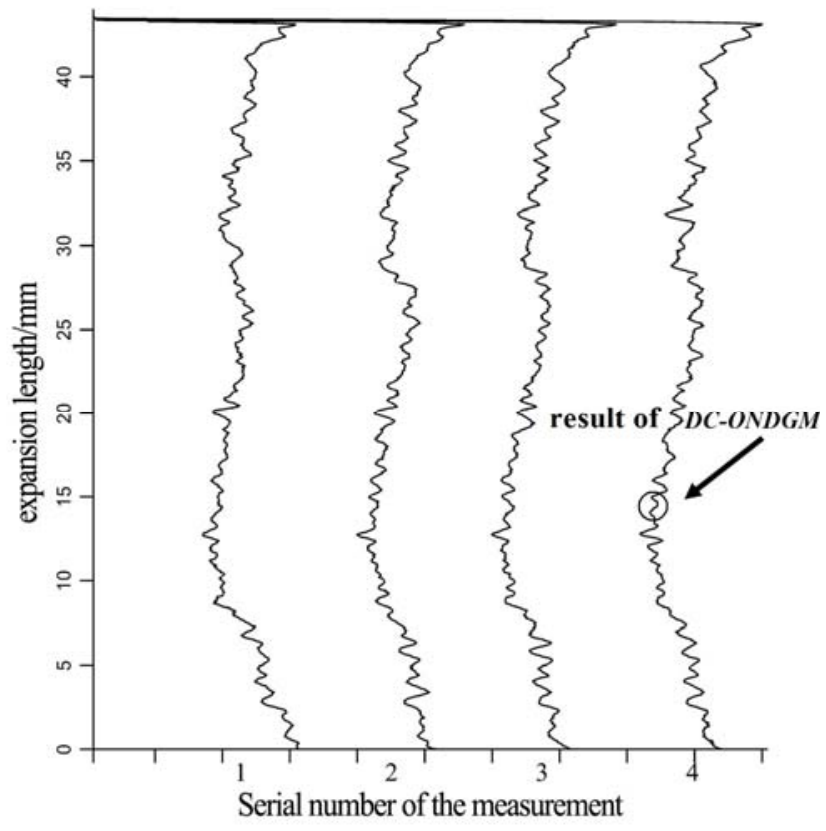

(b). deviation curve processed by DC-ONDGM model

Fig.7. Deviation curves after the outlier processing

Table 2. Evaluation results $(\mu \mathrm{m})$

\begin{tabular}{cccc}
\hline & $F_{\alpha}$ & $f_{f_{\alpha}}$ & $\pm f_{H \alpha}$ \\
\hline original evaluation & -4.4 & -3.6 & -1.8 \\
$\begin{array}{c}\text { evaluation processed by DC- } \\
\text { NDGM }\end{array}$ & -2.8 & -2.4 & -1.5 \\
$\begin{array}{c}\text { evaluation processed by DC- } \\
\text { ONDGM }\end{array}$ & -1.3 & -1.3 & -0.5 \\
actual evaluation & -1.3 & -1.3 & -0.5 \\
\hline
\end{tabular}

Overall, results from the analysis suggest that the DCONDGM model can automatically detect and correct the outliers of the deviations obtained from tooth profiles, so it can improve the measurement precision of the tooth profiles. The effect of the DC-ONDGM model is superior to that of the DC-NDGM model.

\section{CONCLUSIONS}

This paper proposes a preprocessing method for the original deviations of tooth profiles according to the characteristics of the outliers from the tooth profiles and the features of the $\operatorname{NDGM}(1,1)$ model, and establishes a model of outlier detection and correction for the deviations of tooth profiles. The conclusions are as follows.

1). The adjacent variance ratio is a constant and a preprocessing method for the original deviations based on invariant adjacent variance ratio is proposed. The results show that the precision of $\operatorname{NDGM}(1,1)$ is decreased for the outliers, and the precision of the $\operatorname{ONDGM}(1,1)$ model is enhanced by the preprocessing method of the modeling data when the modeling data is abnormal.

2). The DC-ONDGM model automatically detects and corrects the outliers of the deviations obtained from tooth profiles, so it improves the measurement precision of the tooth profiles. The effect of DC-ONDGM model is superior to that of the DC-NDGM model.

\section{ACKNOWLEDGMENT}

This work is jointly supported by the National Nature Science Foundation of China (NO.61108073) and Technology Program of the Educational Office of Hei Longjiang Province in China (No.11553016).

\section{REFERENCES}

[1] Dočekal, A., Dynybyl, V., Kreidl, M., Šmíd, R., Žák, P. (2008). Localization of the best measuring point for gearwheel behaviour testing using group of adaptive models evolution. Measurement Science Review, 8 (2), 42-45.

[2] Takeoka, F., Komori, M., Kubo, A. et al. (2009). High-precision measurement of an involute artefact by a rolling method and comparison between measuring instruments. Measurement Science and Technology, 20, art. no. 045105.

[3] Yang, J., Arai, Y., Gao, W. (2009). Rapid measurement of involute profiles for scroll compressors. Measurement Science Review, 9 (3), 6769.

[4] Lou, Z., Wang, L. (2010). Adjustment of the measurement point's position in a double-disc instrument for measuring an involute. Metrologia, 47, 583-584.

[5] Frazer, R.C., Bicker, R., Cox, B. et al. (2004). An international comparison of involute gear profile and helix measurement. Metrologia, 41, 12-13.

[6] Kamenský, M., Kováč, K. (2011). Correction of ADC errors by additive iterative method with dithering. Measurement Science Review, 11 (1), 15-18. 
[7] Barbato, G., Genta, G., Germak, A., Levi, R., Vicario, G. (2012). Treatment of experimental data with discordant observations: Issues in empirical identification of distribution. Measurement Science Review, 12 (4), 154-159.

[8] Ali, S.H.R. (2010). Probing system characteristics in coordinate metrology. Measurement Science Review, 10 (4), 121-129.

[9] Zhang, F., Qu, X. (2012). Fusion estimation of point sets from multiple stations of spherical coordinate instruments utilizing uncertainty estimation based on Monte Carlo. Measurement Science Review, 12 (2), 40-45.

[10] Miao, Y., Su, H., Gang, R., Chu, J. (2009). Industrial processes: Data reconciliation and gross error detection. Measurement and Control, 42 (7), 209-210.

[11] Koufakou, A., Georgiopoulos, M. (2010). A fast outlier detection strategy for distributed highdimensional data sets with mixed attributes. Data Mining and Knowledge Discovery, 20 (2), 259-289.

[12] Wang, Z., Wang, Q., Wang, X. (2011). A novel method of gross error identification in non-diffracting beam triangulation measurement system. In Fourth International Seminar on Modem Cutting and Measurement Engineering, 10-12 December 2010. SPIE, Vol. 7997, 1-6.

[13] Qin, P., Shen, Y., Wang, Z. (2006). Grey evaluation of non-statistical uncertainty in multidimensional precision measurement. International Journal of Advanced Manufacturing Technology, 31, 539-540.

[14] Zhang, Y., Yang, J., Jiang, H. (2012). Machine tool thermal error modeling and prediction by grey neural network. International Journal of Advanced Manufacturing Technology, 59, 1065-1066.

[15] Gao, Y., Wang, Z., Tao, Z., Lo, C. (2003). Estimation of non-statistical uncertainty in precision measurement using grey system theory. International Journal of Advanced Manufacturing Technology, 22, 271-272.
[16] Meng, H., Zhu, L., Chen, Q. (2010). Detection of abnormal data during dynamic measurement of discontinuous surfaces. In Sixth International Symposium on Precision Engineering Measurements and Instrumentation, 8-11 August 2010. SPIE, Vol. 75440, 1-6.

[17] Shi, Z., Zhu, L., Dong, M. (2003). Grey detection and replacement of unwanted asperities in dynamic measurement of discontinuous curves. Journal of Beijing University of Technology, 29 (1), 15-18.

[18] Wang, Z., Gao, Y., Qin, P. (2002). Detection of gross measurement errors using the grey system method. International Journal of Advanced Manufacturing Technology, 19, 801-804.

[19] Ke, H., Chen, Y., Wu, J. (2008). New distinguishing model for gross error based on $\operatorname{GM}(1,1)$ model. Systems Engineering and Electronics, 30 (10), 20052006.

[20] Lou, Z., Wang, L. (2008). Research on reference level involute testing theory and technology. $\mathrm{PhD}$ thesis, Dalian University of Technology, China.

[21] Xie, N., Liu, S. (2009). Discrete grey forecasting model and its optimization. Applied Mathematical Modeling, 33, 1173-116.

[22] Liu, S., Lin, Y. (2005). Grey Information: Theory and Practical Applications (1 th ed.). Springer-Verlag.

[23] Yao, T., Forrest, J., Gong, Z. (2012). Generalized discrete GM $(1,1)$ model. Grey Systems: Theory and Application, 2 (1), 4-12.

[24] Zhao, J., Yang, S., Xin, L. (2011). Stepwise ratio GM $(1,1)$ model for image denoising. Measurement Science Review, 11 (1), 36-44.

Received August 14, 2012. Accepted April 2, 2013. 